\title{
Efficacy of the EGFr inhibitor Iressa on development of chemically-induced urinary bladder cancers: Dose dependency and modulation of biomarkers
}

\author{
RONALD A. LUBET ${ }^{1}$, YAN LU ${ }^{2}$, ANN M. BODE ${ }^{3}$, MING YOU $^{2}$, ZOE M. VERNEY ${ }^{4}$, VERNON E. STEELE ${ }^{1}$, \\ REID TOWNSEND $^{3}$, M. MARGARET JULIANA ${ }^{5}$ and CLINTON J. GRUBBS ${ }^{6}$
}

\begin{abstract}
${ }^{1}$ Division of Cancer Prevention, National Cancer Institute, 6130 Executive Blvd., Bethesda, MD 20852; ${ }^{2}$ Department of Surgery, Washington University School of Medicine, 660 South Euclid Avenue, St. Louis, MO 63110; ${ }^{3}$ Hormel Institute, University of Minnesota, 801 16th Avenue, Austin, MN 55912; Departments of ${ }^{4}$ Dermatology, ${ }^{5}$ Genetics and

${ }^{6}$ Surgery, University of Alabama at Birmingham, 1530 3rd Avenue South, Birmingham, AL 35294, USA
\end{abstract}

Received January 10, 2011; Accepted February 8, 2011

DOI: 10.3892/or.2011.1200

\begin{abstract}
The effects of the EGFr inhibitor Iressa on development of urinary bladder cancers induced by hydroxybutyl(butyl) nitrosamine $(\mathrm{OH}-\mathrm{BBN})$ in rats were examined. Iressa treatment (4.5 or $1.5 \mathrm{mg} / \mathrm{kg} \mathrm{BW/day)} \mathrm{beginning} \mathrm{one} \mathrm{week} \mathrm{after} \mathrm{the} \mathrm{last}$ dose of OH-BBN decreased the occurrence of large (>200 mg) bladder cancers at termination of the study by 75 and $52 \%$, respectively. Treatment with Iressa (10 mg/kg BW/day) beginning one week or three months (delayed initiation) after the last dose of $\mathrm{OH}-\mathrm{BBN}$ also significantly increased tumor latency and decreased the incidence of palpable bladder cancers. In the delayed initiation study, microscopic cancers already existed when treatment was initiated; implying that the effects of Iressa occur late in tumor progression. Potential pharmacodynamics and/or efficacy biomarkers modulated by short-term exposure (5 day) to Iressa (10 mg/kg BW/day) were determined in palpable bladder lesions by using three different approaches: i) direct immunohistochemical examination of EGFr related proteins; which showed that phosphorylated EGFr, AKT and ERK were significantly decreased; ii) measurement of protein expression by two dimensional gel electrophoresis and tandem mass spectrometry. This showed that the Annexin A2, MAP kinase kinase and nucleolin (all proteins associated with the VEGF pathway) were decreased in treated tumors; and iii) measurement of gene expression determined in gene microarrays demonstrated that numerous pathways were markedly altered by Iressa treatment. In particular, cell cycle genes related to the anaphase protein complex (APC) pathway,
\end{abstract}

Correspondence to: Dr Ronald A. Lubet, National Cancer Institute, Executive Plaza North, Suite 2110, 6130 Executive Blvd., Bethesda, MD 20852, USA

E-mail: lubetr@mail.nih.gov

Key words: hydroxybutyl(butyl)nitrosamine, epidermal growth factor receptor inhibitors, urinary bladder cancer including CDC 20, cyclin B1, BUB1 and both of the Aurora kinases, were significantly decreased.

\section{Introduction}

The $\mathrm{OH}-\mathrm{BBN}$ induced urinary bladder cancer model has been used to screen for potential chemopreventive agents $(1,2)$. The model yields invasive bladder cancers with most tumors being pathologically classified as transitional cell carcinomas (TCC). The resulting tumors show various protein changes; e.g., increased levels of survivin and GST Pi, and decreased levels of FHIT (3). These changes are often observed in human bladder tumors as well. More recently, we have examined gene expression in these tumors by microarray analysis, and have found a wide variety of genes to be overexpressed; including cyclin D1, PCNA, the calcium binding proteins S-100 (4,5), Annexin and numerous genes associated with the EGFr pathway (4). In fact, a recent paper implied that the tumors which form in the rat urinary bladder cancer model are similar to invasive bladder cancers in humans when evaluated by gene arrays (5).

Among the genes found to be overexpressed were those associated with the EGFr pathway. Previous studies in humans have shown overexpression of phosphorylated EGFr in many urinary bladder tumors $(6,7)$. These small molecule inhibitors (Iressa and erlotinib) function as competitive inhibitors of EGFr autophosphorylation at the AMP binding site and may indirectly affect downstream activities in the signaling cascade, including phosphorylation of AKT and ERK (8). We, therefore, recently tested the ability of the small molecule EGFr competitive inhibitor Iressa to prevent urinary bladder tumor formation when administered either immediately following the last dose of $\mathrm{OH}-\mathrm{BBN}$, or 3 months following the last dose of $\mathrm{OH}-\mathrm{BBN}$ when microscopic lesions, including TCC, already existed (1). It was found that Iressa $(10 \mathrm{mg} / \mathrm{kg}$ BW/day, by gavage) was highly effective at either time-point; suggesting that the EGFr inhibitor was effective late during the cancer progression process. In the present studies, these 
findings were expanded to investigate the chemopreventive dose response of Iressa in this model, and to examine potential biomarkers that might give further insight into its mechanism of action. Also, the studies offer potential pharmacodynamic or efficacy biomarkers relevant to treatment with EGFr inhibitors in clinical trials. All biomarkers were examined in rats bearing large urinary bladder cancers that were treated for 5 days with Iressa. Three different approaches were employed in the biomarkers studies: i) specific biomarkers directly associated with inhibition of EGFr, including phosphorylated EGFr, AKT and ERK; ii) non-specific proteins whose expressions were altered as assessed by employing 2-D gel electrophoresis and tandem mass spectroscopy; and iii) non-specific alterations in gene expression employing RNA microarray analyses. Gene changes were examined both for altered expression of specific pathways, as well as identifying a wider group of genes whose expression was significantly altered. It was found that expression of cell cycle related genes were altered, including various cyclins, BUB1 and both Aurora kinases. These various modulated biomarkers may serve as pharmacodynamic and/ or efficacy indicators for treatment with this class of agents in clinical trails.

\section{Materials and methods}

For all studies, female Fischer-344 rats were obtained from Harlan Sprague-Dawley, Inc. (Indianapolis, IN). OH-BBN was purchased from TCI America (Portland, OR) and administered by gavage $(150 \mathrm{mg} /$ dose, $2 \mathrm{x} /$ week for 8 weeks) beginning when the rats were 56 days of age. All animal experiments were conducted in facilities at the University of Alabama at Birmingham following the procedures approved by the Institutional Animal Care and Use Committee. Iressa was supplied by the National Cancer Institute/Division of Cancer Prevention Chemical Repository.

Chemopreventive studies. For the dose-dependent study, Iressa treatment (either 4.5 or $1.5 \mathrm{mg} / \mathrm{kg}$ BW/day) was initiated one week after the final $\mathrm{OH}-\mathrm{BBN}$ treatment (115 days of age). Iressa was administered by gavage daily (vehicle was ethanol: polyethylene glycol 400; 10:90; v/v). Each gavage volume was $0.5 \mathrm{ml}$. As previously reported (1), treatment with Iressa at a higher dose (10 mg/kg BW/day) was also given, but was initiated either 1 week or 3 months after the final $\mathrm{OH}-\mathrm{BBN}$ treatment (rats were 195 days of age at time of initial treatment). All studies were terminated at 8 months after the initial carcinogen treatment. All bladder cancers were confirmed by histological evaluation. All rats were weighed 1x/week and palpated for urinary bladder tumors $2 \mathrm{x} /$ week as previously described $(1,9)$. Rats were sacrificed when they developed a large bladder mass or at termination of the study. Individual weights of the urinary bladders plus lesions in each group were compared using Wilcoxon rank analysis. Differences between the incidence of large bladder tumors (weight of bladder plus lesions $>200 \mathrm{mg}$ ) in the various groups were analyzed by $\chi^{2}$ analysis.

\section{Biomarker studies}

Generation of tissues. OH-BBN treated rats were allowed to develop a palpable lesion and were then treated for 5 days with Iressa (10 mg/kg BW/day) or with the vehicle. At sacrifice, the tumor was divided and either snap frozen in liquid nitrogen or placed in Zamboni's fixative.

Two-dimensional difference gel electrophoresis and image analysis of bladder cancers. Bladder tumors were prepared in a sample buffer (30 mM Tris- $\mathrm{HCl} \mathrm{pH} 8.5,7 \mathrm{M}$ urea, $2 \mathrm{M}$ thiourea, 4\% CHAPS) and labeled with 400 pmol of Cy2, Сy3 or Cy5. Pools were prepared by mixing equal protein amounts of each sample following labeling. All samples were then equilibrated into immobilized $\mathrm{pH}$ gradient (IPG) strips under $100 \mathrm{~V}$ followed by isoelectric focusing using a maximum of 10,000 focusing volts (Protean IEF cell, Bio-Rad). After focusing, proteins were reduced with TCEP $(10 \mathrm{mM})$ and alkylated with iodoacetamide $(20 \mathrm{mM})$. The IPG strip was then layered on $10 \%$ polyacrylamide gels followed by SDS-PAGE separation. Samples were imaged with a Typhoon Imager (GE Healthcare) using specific excitation/emission wavelengths: 488/520 nm for Cy2, 520/580 nm for Cy3, 620/670 nm for Cy5. The DeCyder (ver. 6.5) software tools (GE Healthcare) were used for image analysis. The DIA (differential in-gel analysis) module was used to align and normalize images within each gel. Spots with slopes $>1.1$, areas $<100$, volumes $<10,000$ and peak heights $<100$ were excluded. The DIA module calculated abundance ratios using a normalization algorithm that was applied as previously described (10). For comparative pairwise analyses of gel features across different physical gels, the DIA data sets were analyzed using the BVA (biological variation analysis) module. Spot volumes were converted to ratios of a pooled internal standard. Using the extended data analysis (EDA) module, a t-test was performed to determine the statistical significance of ratios for each gel feature.

Protein identification of gel features using tandem mass spectrometry. Gel features were excised robotically (ProPic, Genomic Solutions) using a triangulation algorithm implemented with in-house software. The gel pieces were digested in situ with trypsin (11). Samples were processed and analyzed using nano-LC-FTMS/MS as previously described (12). The 'raw' files were processed using MASCOT Distiller, version 2.1.1.0 (Matrix Science, Oxford, UK) with the following settings: i) MS processing, 200 data points per Da; no aggregation method; maximum charge state, +8 ; minimum number of peaks, 1. ii) MS/MS processing, 200 data points per Da; time domain aggregation method enabled; minimum number of peaks, 10; precursor charge and $m / z$, try to re-determine from the survey scan (tolerance, $2.5 \mathrm{Da}$ ); charge defaults, $+2 /+3$; maximum charge state, +2 . iii) Time domain parameters, minimum precursor mass, 700; maximum precursor mass, 16,000 ; precursor $\mathrm{m} / \mathrm{z}$ tolerance for grouping, 0.1 ; maximum number of intermediate scans, 5 ; minimum number of scans in a group, 1. iv) Peak picking, maximum iterations, 500; correlation threshold, 0.90; minimum signal-to-noise, 3 ; minimum peak $m / z, 50$; maximum peak $m / z, 100,000$; minimum peak width, 0.001 ; maximum peak width, 2 ; and expected peak width, 0.01. The resulting Mascot generic files (mgf) were exported to MASCOT, ver. 2.1.6. The tandem MS data from the LTQ-FT were searched against National Center for Biotechnology Information non-redundant databases that were downloaded on 2008-11-26 with the following constraints: MS tolerance, $10 \mathrm{ppm}, \mathrm{MS} / \mathrm{MS}$ tolerance, $0.8 \mathrm{Da}$ with fixed 
Table I. Weights of urinary bladders with $\mathrm{OH}-\mathrm{BBN}$ induced tumors in female Fischer-344 rats treated with Iressa.

\begin{tabular}{lcc} 
Treatment $^{\mathrm{a}}$ & ${\text { Urinary bladder }+ \text { tumors }(\mathrm{mg})^{\mathrm{b}}}^{\mathrm{b}}$ & No. of urinary bladder weights $>200 \mathrm{mg}^{\mathrm{c}}$ \\
\hline Iressa, $4.5 \mathrm{mg} / \mathrm{kg} \mathrm{BW} /$ day & $148 \pm 9(55 \% \downarrow)^{\mathrm{d}}$ & $5 / 30(17 \%)^{\mathrm{d}}$ \\
Iressa, $1.5 \mathrm{mg} / \mathrm{kg} \mathrm{BW/day}$ & $192 \pm 14(42 \% \downarrow)$ & $10 / 30(33 \%)^{\mathrm{d}}$ \\
None & $332 \pm 39$ & $20 / 29(69 \%)$
\end{tabular}

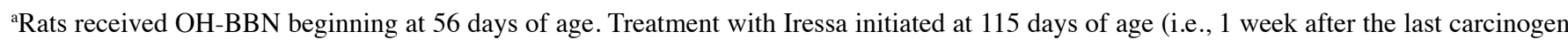

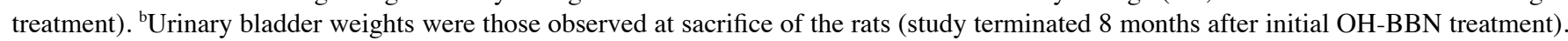
Values are mean \pm SEM (number in parenthesis is percent decrease from controls). 'Values represent the number of rats with urinary bladder weights $>200 \mathrm{mg}$ divided by the number of rats in that group. ${ }^{\mathrm{d} V}$ Values were significantly different $(\mathrm{p}<0.05)$ from controls.

modifications of cysteine residues (carbamidomethylation) and variable oxidation of methionine residues. The resulting DAT files were imported into Scaffold, ver. 2.0 (Proteome Software, Portland, OR) to identify proteins with $>95 \%$ confidence and to determine the spectral counts for each protein. The network analysis was generated using Ingenuity Pathways Analysis software, ver. 6.

IHC detection of phosporylated proteins. Immunohistochemical detections of phosphorylation of EGFr (Tyr1173), ERKs, Akt (Ser473), total EGFr and Ki67 were performed. Bladder tumors were excised and drop fixed in Zamboni's fixative $[0.03 \%$ picric acid $(\mathrm{w} / \mathrm{v})$ and $2 \%$ paraformaldehyde $(\mathrm{w} / \mathrm{v})]$ for $48 \mathrm{~h}$ at $5^{\circ} \mathrm{C}$ and then transferred to a $20 \%$ sucrose solution with $0.05 \%$ sodium azide in PBS for storage at $5^{\circ} \mathrm{C}$. Processing and staining of tumors were carried out according to a published procedure (13). Whole tumors were cryosectioned into $80 \mathrm{~mm}$ sections. Floating sections were incubated first with the primary antibody, and then incubated in 1:10,0000 anti-goat IgG, conjugated to $\mathrm{Cy} 3$ (red color) or Cy 5 (blue color) (Jackson Immuno Research). Washed samples were mounted in agar, dehydrated in ethanol, cleared with methyl salicylate and mounted in DEPEX (Electron Microscopy Science). Optical sections were captured by laser scanning confocal microscopy.

RNA isolation and amplification. Bladder tumors were excised and frozen at $-80^{\circ} \mathrm{C}$ for subsequent molecular assays. A portion of each tumor was fixed, processed for paraffin embedding, cut into $5-\mu \mathrm{m}$ sections, and mounted for hematoxylin and eosin (H\&E) staining. All urinary bladder tumors used in this study were diagnosed as bladder cancers. Total RNA from bladder tumors treated with Iressa were isolated by TRIzol (Invitrogen, Carlsbad, CA) and purified using the RNeasy Mini Kit and RNase-free DNase Set (Qiagen, Valencia, CA) according to the manufacturer's protocol. In vitro transcription-based RNA amplification was then performed on each sample. cDNA for each sample was synthesized using a Superscript cDNA Synthesis Kit (Invitrogen) and a T7-(dT)24 primer: 5'-GGCCAGTGAATTGTAATACGACT CACTATAGGGAGGCGG-(dT)24-3'. The cDNA was cleaned using phase-lock gel phenol/chloroform extraction (Fisher Scientific). The biotin-labeled cRNA was then transcribed in vitro from cDNA using a BioArray High Yield RNA
Transcript Labeling Kit (ENZO Biochem, New York, NY) and purified, again using the RNeasy mini kit.

Affymetrix GeneChip probe array analysis. The labeled cRNA was applied to the Affymetrix Rat Exon 1.0 ST Array (Affymetrix, Santa Clara, CA) according to the manufacturer's recommendations. Gene-level signal estimates for the CEL files from the platform Rat Exon 1.0 ST Array were derived by quantile sketch normalization using Iterplier algorithm, as implemented with Expression Console v1.1.1 (http:// www.affymetrix.com/products services/software/specific/ expressionconsolesoftware.affx). A two-sample student t-test was used to identify differentially expressed genes (DEGs) between groups. To adjust for the multiple testing in the study of high-dimensional microarray data, local false discovery rate (LFDR) were estimated which was implemented in $\mathrm{R}$ package fdrtool (http://www.r-project.org/) (14). The DEGs were defined as genes with LFDR $<0.05$ and fold change $>1.5$ between two groups.

After identification of DEGs, they are inputted into gene set enrichment analysis (GSEA). GSEA is a computational method that determines whether a set of genes shows statistically significant differences in expression between two biological states. This has proved successful in discovering molecular pathways involved in human diseases (http://www.broad.mit. edu/gsea). Using the Kolmogorov-Smirnov statistic, GSEA assesses the degree of 'enrichment' of a set of genes (e.g., a pathway) in the entire range of the strength of associations with the phenotype of interest. It was used to identify a priori defined sets of genes that were differentially expressed $(15,16)$. We used curated gene sets (c2) which contain genes in certain molecular pathways, and GO gene sets (c5) which consist of genes annotated by the same GO terms in the Molecular Signature Database (MSigDB, http://www.broad.mit.edu/ gsea/msigdb/msigdb_index.html). Because of small sample sizes, GSEA with gene set permutation option was performed. Selected gene sets identified from GSEA were then visualized with MetaCore $^{\text {TM }}$ (http://www.genego.com/). Hierarchical clustering was performed as follows: For the selected genes, expression indexes were transformed across samples to an $\mathrm{N}$ $(0,1)$ distribution using a standard statistical Z-transform. These values were inputted into the GeneCluster program of Eisen et al (17), and genes were clustered using average linkage and correlation dissimilarity. 


\section{Results}

Effect of Iressa in prevention studies. Iressa was evaluated at two dose levels to determine the lowest effective dose in preventing the formation of urinary bladder cancers. Iressa administered at doses of 4.5 and $1.5 \mathrm{mg} / \mathrm{kg} \mathrm{BW} /$ day, $7 \mathrm{x} /$ week, decreased the number of large urinary bladder cancers (weights of total bladder plus tumors $>200 \mathrm{mg}$ ) from $69 \%$ in the controls to 17 and $33 \%$, respectively (Table I). Furthermore, when the time of appearance of the palpable urinary bladder tumors was graphed (Fig. 1A), the $4.5 \mathrm{mg} / \mathrm{kg} \mathrm{BW} /$ day dose was highly effective both in increasing tumor latency and in preventing the development of large tumors. A previous study from our laboratory (1) had demonstrated that a higher dose of Iressa $(10 \mathrm{mg} / \mathrm{kg}$ $\mathrm{BW} /$ day) was even more effective in preventing the development of large tumors (Fig. 1B and C). This dose of Iressa was highly effective in increasing latency and decreasing the incidence of palpable tumors when administered beginning 1 week or 3 months after the last dose of OH-BBN. The efficacy seen in the late administration is particularly interesting because at the later point $70 \%$ of rats already had invasive carcinomas (Fig. 1C).

Effects of EGFr inhibitors on tumor related biomarkers. Based on the striking efficacy following late administration of Iressa, we examined the effects of limited treatment for 5 days on palpable bladder cancers with Iressa (10 mg/kg BW/day). Three different approaches were employed to identify potential biomarkers.

Examination of EGFr pathway related biomarkers (Fig. 2). The primary focus of these studies was to examine the effect of Iressa on proteins associated with the EGFr pathway including EGFr and its downstream effector proteins AKT and ERK. Using immunohistochemistry and confocal microscopy, it was found that levels of phosphorylated EGFr (Tyr1173), Akt (Ser473) and ERKs were decreased by 50,75 and 47\%, respectively, in bladder cancers from rats receiving Iressa. These differences were found to be significant, and demonstrate the direct effects of Iressa on the EGFr pathway.

Examination of the effects of Iressa on protein expression employing 2D gel (Fig. 3). In these studies, protein expression levels from Iressa treated and control tumors were measured by labeling with fluorometrically distinct cyanine dyes (18). In the image shown in Fig. 3A, the green colored gel features indicate proteins that are decreased in the treated samples. Of the 1500 gel features, roughly 200 were found to be changed outside of the $95 \%$ confidence percentile. The differential gel features were excised, digested with trypsin in situ, and the proteins identified from the fragmentation spectra of the peptides.

A three dimensional view of two proteins associated with the VEGF pathway [Annexin A2 and MAP kinase kinase (MAP2K)] were shown to be decreased 2.3- and 4.5-fold, respectively, in the tumors from treated rats. The complete set of differentially expressed proteins was analyzed for network and pathway associations using Ingenuity Pathways Analysis. The VEGF pathway exhibited decreases in Annexin A2 and MAP kinase kinase (Fig. 3B), but also showed decreases in nucleolin (NCL) and the small GTPase Rho A; both of which also function in the VEGF pathway.

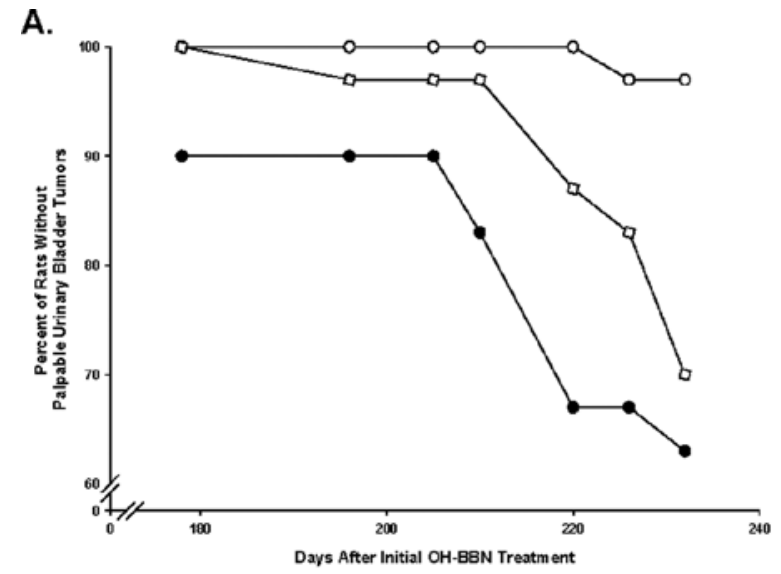

B.

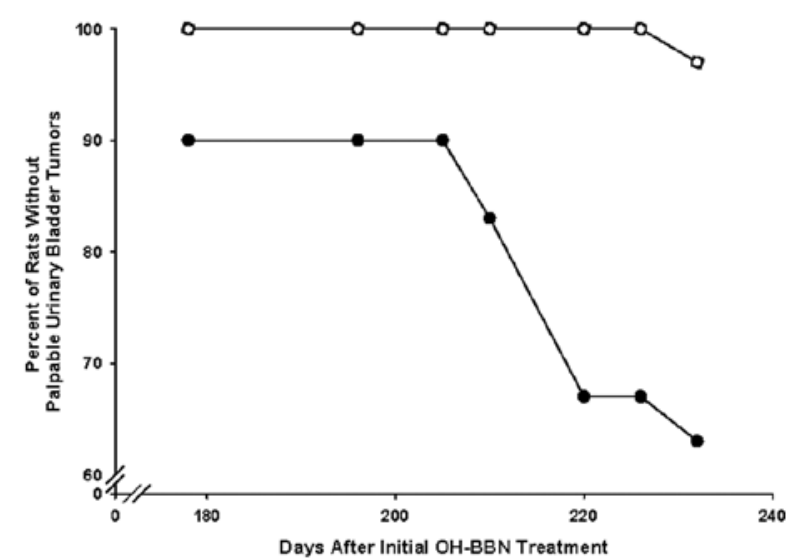

C.

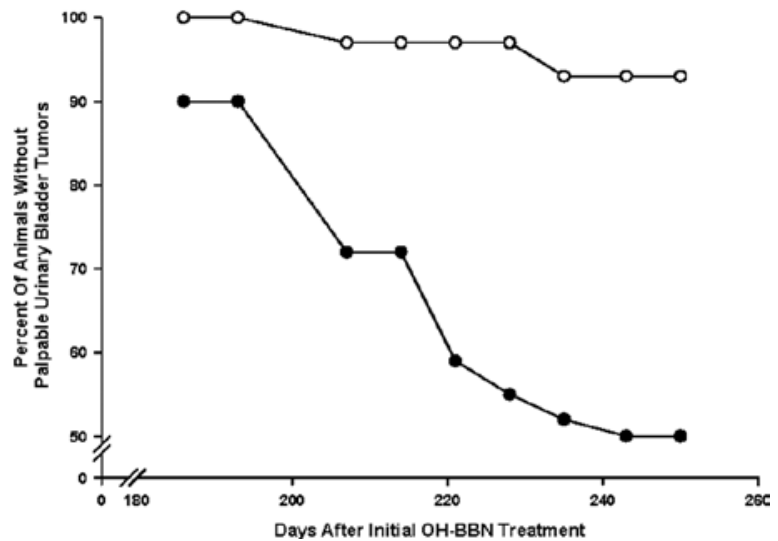

Figure 1. Effects of Iressa on the time of appearance of palpable urinary bladder tumors in female Fischer-344 rats. (A) Iressa (4.5 and $1.5 \mathrm{mg} / \mathrm{kg} \mathrm{BW/}$ day) was administered from one week after the final $\mathrm{OH}-\mathrm{BBN}$ treatment until the end of the study. $\bigcirc$, Iressa $4.5 \mathrm{mg} / \mathrm{kg} \mathrm{BW} /$ day; $\square$, Iressa $1.5 \mathrm{mg} / \mathrm{kg} \mathrm{BW/}$ day; •, control. (B) Effects of high dose Iressa (10 mg/kg BW/day) on time of appearance of palpable urinary bladder tumors in female Fischer-344 rats (1). Iressa was started one week after the final $\mathrm{OH}-\mathrm{BBN}$ treatment. $\bigcirc$, Iressa $10 \mathrm{mg} / \mathrm{kg} \mathrm{BW} /$ day; $\bullet$, control (C). Iressa was started 3 months after the final $\mathrm{OH}-\mathrm{BBN}$ treatment. $\bigcirc$, Iressa $10 \mathrm{mg} / \mathrm{kg} \mathrm{BW} /$ day; $\bullet$, control.

Examination of the effects of Iressa on gene expression employing oligonucleotide microarrays (Figs. 4 and 5). In 
A.

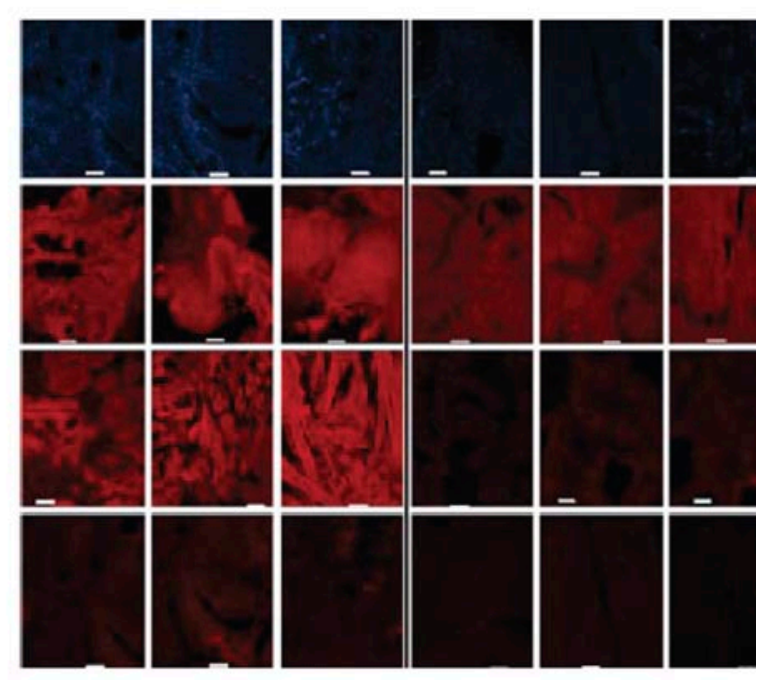

B.

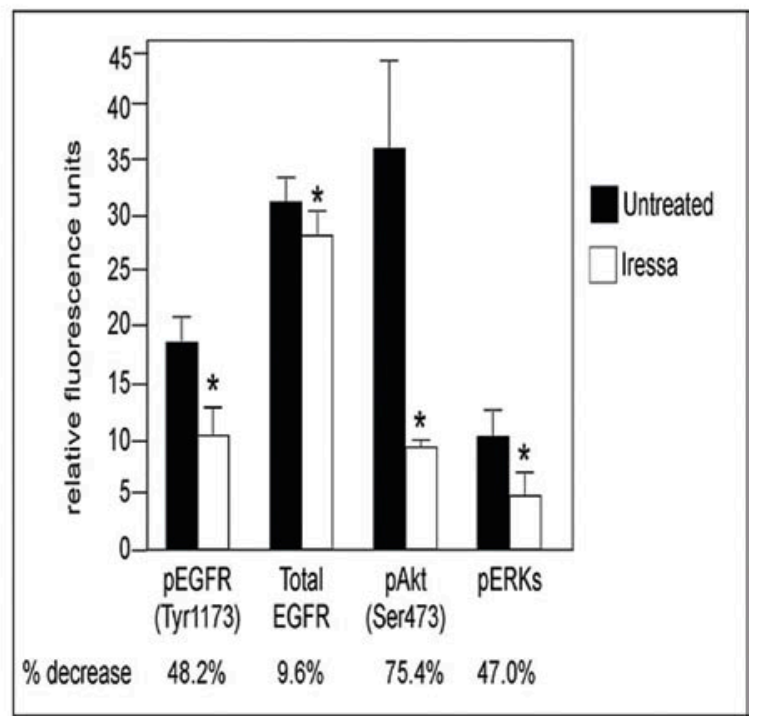

Figure 2. Immunostaining and quantitation of pEGFR, total EGFR, pAKT and pERKs in urinary bladder tumors from untreated or Iressa treated rats $(10 \mathrm{mg} / \mathrm{kg}$ BW/day for 5 days). (A) Representative bladder cancers from untreated rats (left 3 columns) or Iressa treated rats (right 3 columns). Slides were immunolabeled with antibodies to detect specific proteins (top row, pEGFR; second from top, EGFR; third from top, pAKT; bottom, pERK). The image is a z-projection created from $301 \mu$ sections using laser confocal microscopy (Nikon Cls Confocal Spectral Imaging System, Nikon Instrument Company) with a CFI Plan Fluorx 20x objective. (B) The light intensity of three different cancers was estimated using the Image J computer program; data shown as mean $\pm \mathrm{SD}$. Asterisk indicates decreased expression, $\mathrm{P}<0.05$.

these studies, the effects of Iressa on gene expression by employing oligonucleotide microarrays were determined. As shown in Fig. 5, there were an extremely large number of genes whose expressions were altered when comparing bladder cancers treated short-term with Iressa and control bladder cancers. The resulting gene expression changes were examined using gene pathway analysis, and genes in several pathways were identified. The gene expression pathway analysis (Fig. 4) and accompanying expression levels (Table II) of genes associated with cell cycle pathways [specifically, the anaphase protein complex (APC)] are presented. Expression of various genes, including cyclins $\mathrm{B}$ and D, CDC20, BUBR1, BUB1, securin and Aurora B, were all substantially decreased in Iressa treated vs control bladder tumors. When expression of the genes coding for E cadherin and vimentin were compared in normal bladder epithelium and bladder tumors, Cdh1 was modestly down-regulated in bladder tumors (LFDR=0.003 and fold change $=-1.42$ ); no difference in expression of Vim was found.

\section{Discussion}

Studies were performed to examine the efficacy of an EGFr inhibitor in the $\mathrm{OH}-\mathrm{BBN}$ induced model of urinary bladder cancers, as well as to examine potential expression changes which might prove applicable as pharmacodynamic or efficacy biomarkers in clinical trials using this class of agents. The tumors that develop in the $\mathrm{OH}-\mathrm{BBN}$ induced model are primarily transitional cell or mixed cell carcinomas as defined by histopathology. In addition, they exhibit many changes found in human bladder cancers $(3,4)$, including increased levels of survivin, GST Pi, Annexin, various S-100 proteins, and decreased levels of FHIT. Recently, we have compared urinary bladder tumors with normal bladder epithelium employing gene arrays (4). Those results showed alterations in a variety of pathways including general overexpression of the genes related to the EGFr pathway.

Based on the fact that EGFr is expressed at higher levels in bladder tumors than in normal bladder epithelium $(6,7)$, both in humans and in rats, the efficacy of Iressa was tested in the OH-BBN bladder cancer model. Iressa treatment initiated at one week following the last dose of OH-BBN yielded a dose-dependent decrease in the formation of urinary bladder cancers. Interestingly, the highest dose employed $(10 \mathrm{mg} / \mathrm{kg}$ BW/day) is approximately $2 \mathrm{x}$ less than the typical human dose; based on a standard rat/human scaling factor (1). It was found that even lower doses of Iressa (4.5 and $1.5 \mathrm{mg} / \mathrm{kg} \mathrm{BW} /$ day) were effective in preventing $\mathrm{OH}-\mathrm{BBN}$ induced bladder tumors. More importantly, the highest dose of Iressa was highly effective in increasing tumor latency when administered beginning three months following the final dose of OH-BBN (Fig. 1C); at a time when $70 \%$ of animals had already developed microscopic invasive bladder lesions (1). Highly significant prevention at this late stage suggests that the effect of Iressa was primarily on the progression stage of the carcinogenic process. Based on these findings, various data with human bladder cancer cell cultures, and the finding that EGFr is overexpressed in many human bladder cancers support the use of an EGFr inhibitor in an adjuvant setting in humans. In fact, the EGFr inhibitors Tarceva (EGFr1) and Lapatinib (EGFr 1 and 2) have undergone small Phase II trials in more advanced bladder cancers, and both appear to show activity $(19,20)$.

The determination of potential biomarkers has multiple objectives. First, it may offer some mechanistic insights into the mode of action of a given agent or class of agents. Although in the case of Iressa, the primary target is clearly defined. Changes in specific pathways [e.g., VEGF (proteomics) or cell cycle (genomics)], may give insights into the molecular mechanism(s) underlying the efficacy of the agent. Second, since these changes are observed at a highly effective dose of Iressa, some of the observed changes may identify biomarkers related to the efficacy of the agent. However, even if these changes are primarily pharmacodynamic (implying pharma- 
A

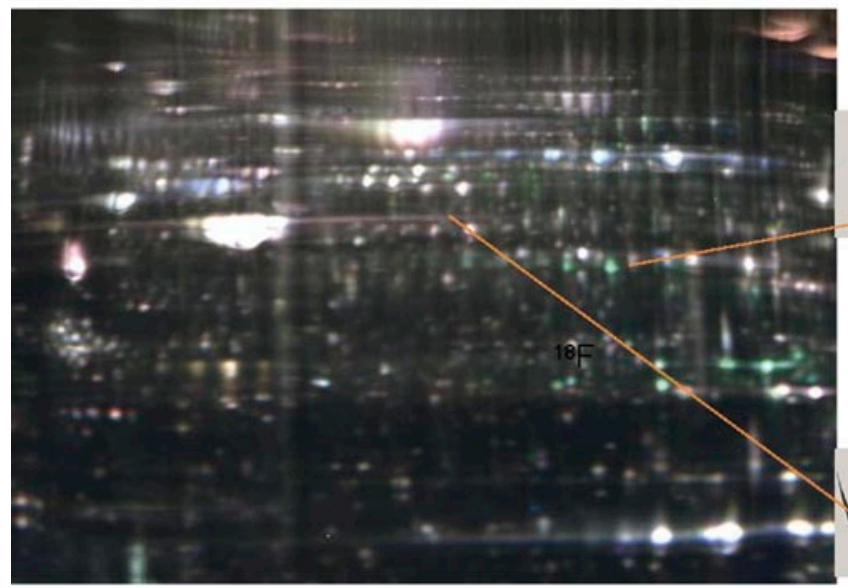

B

Annexin A2
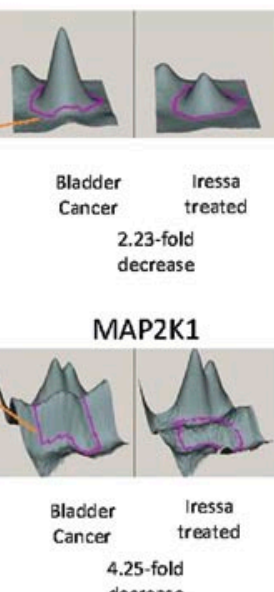

Figure 3. Proteomic analysis of urinary bladder cancers from Iressa-treated and control rats. (A) Two-dimensional difference gel electrophoresis as described in Materials and methods. Green spots are overexpressed in control tumors. Red spots are overexpressed in Iressa-treated tumors. (B) Topological mapping of two individual spots (Annexin A2, MAP2K1) after identification of peptides by mass spectrometry.

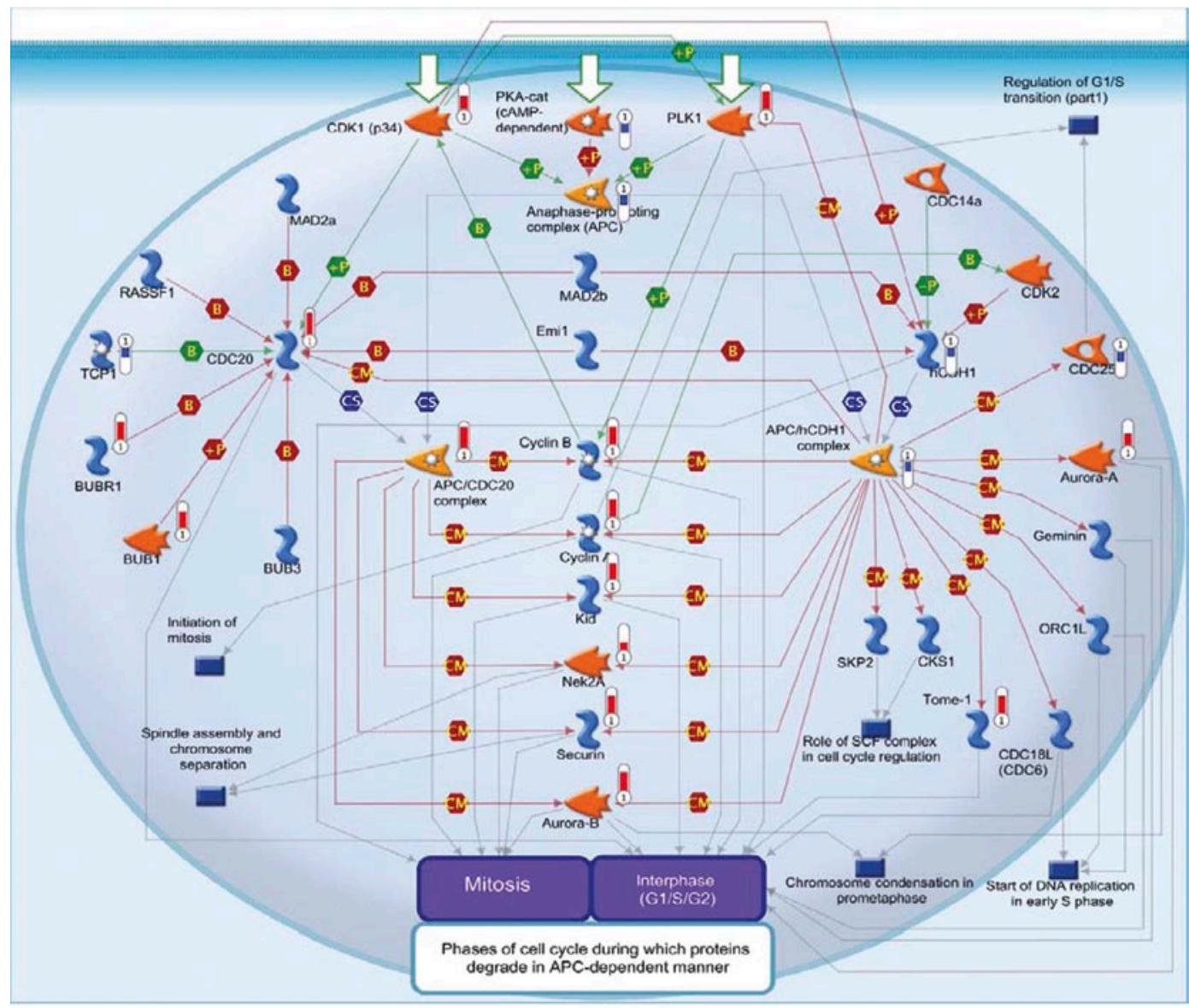

Downregulated by Iressa treatment compared with control bladder tumor $(\mathrm{P}<0.05)$

Upregulated by Iressa treatment compared with control bladder tumor $(P<0.05)$

Figure 4. Effects of Iressa on cell cycle related genes associated with anaphase promoting complex (APC). Alterations in specific genes are based on Affymatrix analysis of gene expression (Materials and methods). 


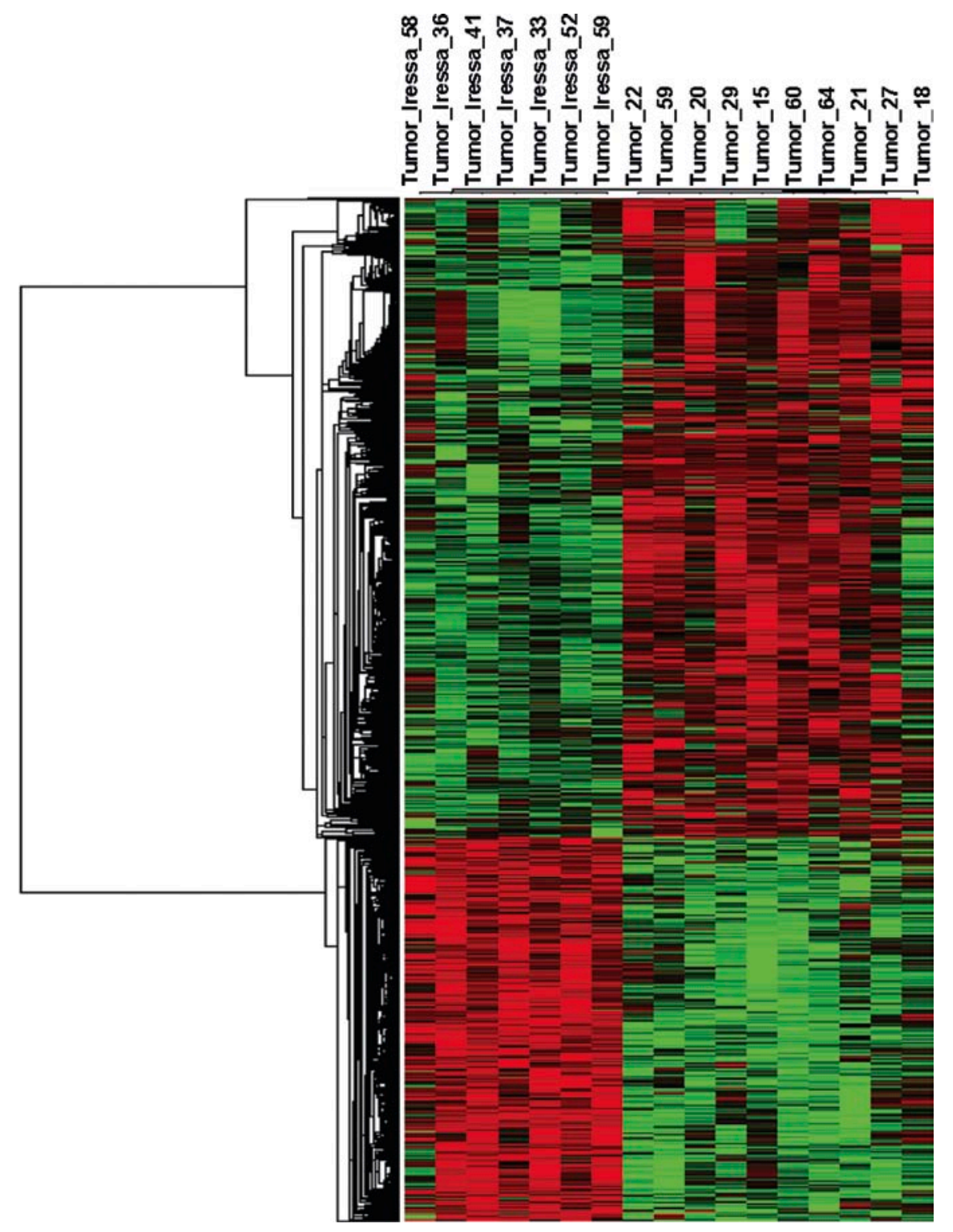

Figure 5. Gene showing changes in expression when comparing cancers from Iressa-treated and control rats. Data based on microarray analysis: red, overexpressed; green, underexpressed; black, average expression.

Table II. Significant changes in gene expression in cell cyle APC related pathway.

\begin{tabular}{llccccc}
\hline & & \multicolumn{2}{c}{ Mean \pm SD } & & \\
\cline { 3 - 5 } Probe set ID & Gene & Control tumor & Treated tumor & P-value & LFDR & Fold change \\
\hline 7290458 & CDC20 & $372 \pm 113$ & $124 \pm 70$ & 0.000116 & 0.0056 & 3.01 \\
7207681 & CCNA2 & $522 \pm 194$ & $198 \pm 162$ & 0.002608 & 0.019895 & 2.64 \\
7203074 & CCNB1 & $425 \pm 124$ & $162 \pm 121$ & 0.000569 & 0.009926 & 2.62 \\
7036458 & PLK1 & $691 \pm 253$ & $249 \pm 177$ & 0.001245 & 0.012326 & 2.77 \\
7244612 & BUB1 & $498 \pm 161$ & $205 \pm 156$ & 0.002007 & 0.015968 & 2.43 \\
7231515 & BUB1B & $359 \pm 128$ & $137 \pm 86$ & 0.00118 & 0.012326 & 2.63 \\
7076550 & PTTG1 & $207 \pm 77$ & $81 \pm 55$ & 0.002172 & 0.015968 & 2.55 \\
7057437 & KIF22 & $278 \pm 84$ & $125 \pm 75$ & 0.001485 & 0.012708 & 2.23 \\
7248426 & AURKA & $255 \pm 66$ & $155 \pm 56$ & 0.005007 & 0.027147 & 1.65 \\
7068974 & AURKB & $352 \pm 131$ & $132 \pm 99$ & 0.001997 & 0.015968 & 2.66 \\
7258359 & CDCA3 & $225 \pm 63$ & $86 \pm 34$ & $9.24 \mathrm{E}-05$ & 0.004977 & 2.63 \\
7218195 & CDC2 & $224 \pm 59$ & $110 \pm 74$ & 0.003072 & 0.020887 & 2.03 \\
\hline
\end{tabular}


cologic/physiologic effects) for the agent, these biomarkers may be important to potential clinical prevention trials, particularly Phase II trials. In examining the biomarker data, one must be aware that although Iressa can block further tumor development (even beginning at a time when established carcinomas exist), it has not been shown that it can cause the regression of existing large lesions. Part of the problem is that we typically identify palpable lesions that are relatively large and that have invaded the underlying tissues. Thus, a method should be developed that will permit the identification of smaller lesions in order to determine whether an EGFr inhibitor is therapeutic in this model.

The first set of potential biomarkers examined was directly related to inhibition of phosphorylated EGFr. Immunohistochemistry showed that Iressa induced significant decreases in amounts of phosphorylated EGFr (Tyr 1173)s, phosphorylated AKT (ser 473) and phosphorylated ERKs. Interestingly, our laboratories have observed modulation of these same parameters in a mammary cancer model in which Iressa was similarly highly effective. In this case, the results were confirmed employing Western blot analyses. These studies parallel a pre-surgical model of cancer that could demonstrate the modulation of the expected targets of the inhibitor (8).

The second biomarker approach looked at potential changes in expression of various proteins of bladder cancers employing a 2D gel approach. Proteins were extracted, labeled with cyanine dyes, separated by 2D electrophoresis, and the images were co-registered with software. The relative abundance of proteins in tumors of Iressa-treated rats can be determined without variability associated with aligning images from multiple $2 \mathrm{D}$ analyses. The verification of the approach is illustrated by the identification of proteins that are well recognized to be associated with the effects of the VEGF pathway in tumor angiogenesis (Fig. 3). Annexin II is a tumor associated protein with a potential role in tumor progression and angiogenesis (21). The decrease in expression of MAPK kinase, a protein associated with angiogeneis (22), suggests downstream effects of EGFr inhibitors. In summary, these 2D gel findings are consistent with previous data indicating that alterations in the VEGF pathway are associated with effects of the EGFr inhibitors (23). These changes, as mentioned above, are probably secondary to the initial inhibition of EGFr phosphorylation. Nevertheless, changes in the angiogenesis pathway may contribute to the overall efficacy that was observed.

Finally, gene expression oligonucleotide arrays were performed to determine which genes exhibit altered expression levels in bladder cancers from Iressa treated rats. Although gene expression is two steps removed from protein function and protein expression, it has many advantages; including the ability to examine simultaneously the widest variety of genes, and to integrate the results into pathways or functional analyses. Numerous pathways were found to be altered (cell cycle, G-protein receptor, DNA damage and DNA repair). The genes associated with the anaphase protein complex pathway that were altered are presented in Table II and Fig. 4. The changes were statistically significant and caused 2- to 4-fold decreases in gene expression. Included among these genes were CCNA2, CDC2, CDC20, cyclin B1 and Aurora kinases A and B. These differences support the finding of substantial decreases in proliferation in Iressatreated vs control bladder cancers (data not shown). The changes also correlate with the high efficacy of Iressa in this model of urinary bladder cancer (Fig. 1A and B). We also examined the expression of $\mathrm{E}$ cadherin (an epithelial cell marker) and vimentin (a mesenchymal cell marker) in normal urinary bladder epithelium and bladder cancers. The rationale being that tumors which have undergone a mesenchymal cell transition are less likely to respond to an EGFr inhibitor. The minimal differences observed in expression of $\mathrm{E}$ cadherin and vimentin likely reflect a limited mesenchymal transition and may suggest that EGFr inhibitors are highly effective; even at the later stages of progression. We feel that these potential biomarkers may be useful in clinical prevention or in adjuvant trials with this class of agents. One would not expect that most or all of the biomarkers from a rat would translate into a human, although the biomarkers directly related to the EGFr phosphorylation pathway (EGFrP, AktP and ERKP) are likely to be relevant across species. Furthermore, more complete analyses of the results from the 2D gels and gene arrays may yield a wide variety of potential biomarkers, of which some significant number are likely to be relevant to humans.

\section{Acknowledgements}

The authors wish to thank Jeanne Hale and Mary Jo Cagle for secretarial and editorial services. Also, thanks are expressed to Tom Morgan, Bonnie Dillion, Emily Meadows, John Houston and Jimmy Anderson for assistance with the animal studies.

\section{References}

1. Lubet RA, Steele VE, Juliana MM and Grubbs CJ: Screening agents for preventive efficacy in a bladder cancer model: study design, endpoints, and gefitinib and naproxen efficacy. J Urol 183: 1598-1603, 2010.

2. Lubet RA, Yang CS, Lee MJ, et al: Preventive effects of polyphenone $\mathrm{E}$ on urinary bladder and mammary cancers in rats and correlations with serum and urine levels of tea polyphenols. Mol Cancer Ther 6: 2022-2028, 2007.

3. Lubet RA, Huebner K, Fong LY, et al: 4-Hydroxybutyl(butyl) nitrosamine-induced urinary bladder cancers in mice: characterization of FHIT and survivin expression and chemopreventive effects of indomethacin. Carcinogenesis 26: 571-578, 2005.

4. Yao R, Yi Y, Grubbs CJ, Lubet RA and You M: Gene expression profiling of chemically induced rat bladder tumors. Neoplasia 9: 207-221, 2007.

5. Williams PD, Lee JK and Theodorescu D: Molecular credentialing of rodent bladder carcinogenesis models. Neoplasia 8: 838-846, 2008

6. Chow NH, Chan SH, Tzai TS, Ho CL and Liu HS: Expression profiles of ErbB family receptors and prognosis in primary transitional cell carcinoma of the urinary bladder. Clin Cancer Res 7: 1957-1962, 2001.

7. Colquhon AJ and Mellon JK: Epidermal growth factor receptor and bladder cancer. Postgrad Med J 78: 584-589, 2002.

8. Baselga J: Targeting the epidermal growth factor receptor: a clinical reality. J Clin Oncol 10: 41-45, 2001.

9. Steele VE, Rao CV, Zhang Y, et al: Chemopreventive efficacy of naproxen and nitric oxide-naproxen in rodent models of colon, urinary bladder, and mammary cancers. Cancer Prev Res 2: 951-956, 2009.

10. Alban A, David SO, Bjorkesten L, Anderson C, Sloge E, Lewis $\mathrm{C}$ and Currie I: A novel experimental design for comparative two-dimensional gel analysis: two dimensional difference gel electrophoresis incorporating a pooled internal standard. Proteomic 3: 36-44, 2003. 
11. Bredemeyer AJ, Lewis RM, Malone JD, Davis AE, Gross J, Townsend RR and Ley TJ: A proteomic approach for the discovery of protease substrates. Proc Natl Acad Sci USA 101: 11785-11790, 2004.

12. King JB, Gross J, Lovly CM, Rohrs H, Piwnics-Worms H and Townsend RR: Accurate mass-driven analysis for the characterization of protein phosphorylation. Study of the human Chk2 protein kinase. Anal Chem 78: 2171-2181, 2006.

13. Wacnik PW, Baker CM, Herron MJ, et al: Tumor-induced mechanical hyperalgesia involves CGRP receptors and altered innervation and vascularization of DsRed2 fluorescent hindpaw tumors. Pain 115: 95-106, 2005.

14. Strimmer K: A unified approach to false discovery rate estimation. BMC Bioinformatics 9: 303, 2008.

15. Mootha VK, Lindgren CM, Eriksson KF, et al: PGC-1alpharesponsive genes involved in oxidative phosphorylation are coordinately down-regulated in human diabetes. Nat Genet 34: 267-273, 2003.

16. Subramanian A, Tamayo P, Mootha VK, et al: Gene set enrichment analysis: a knowledge-based approach for interpreting genome-wide expression profiles. Proc Natl Acad Sci USA 102: $15545-15550,2005$.
17. Eisen MB, Spellman PT, Brown PO and Botstein D: Cluster analysis and display of genome-wide expression patterns. Proc Natl Acad Sci USA 95: 14863-14868, 1998.

18. Unlu M, Morgan ME and Minden JS: Difference gel electrophoresis: a single gel method for detecting changes in protein extracts. Electrophoresis 18: 2071-2077, 2005.

19. Wulfing C, Machiels JP, Richel DJ, et al: A single arm, multicenter, open-label phase 2 study of lapatinib as the second line treatment of patients with locally advanced or metastatic transitional cell carcinoma. Cancer 115: 2881-2890, 2009.

20. Pruthi RS, Nielson M, Heathcote S, et al: A phase II trial of neoadjuvant erlotinib in patients with muscle-invasive bladder cancer undergoing radical cystectomy: clinical and pathological results. BJU Inter 106: 349-356, 2010.

21. Sharma MC and Sharma M: The role of annexin II in angiogenesis and tumor progression: a potential therapeutic target. Current Pharm Design 13: 3568-3575, 2007.

22. Kim LC, Song L and Harua EB: Src kinases as therapeutic targets for cancer. Nat Rev Clin Oncol 6: 587-595, 2009.

23. Kassouf W, Brown GA and Black PC: Is vascular endothelial growth factor modulation a predictor of the therapeutic efficacy of gefitinib for bladder cancer? J Urol 180: 1146-1153, 2008. 